Supplementary data:

\title{
Why All the Fuss about Oxidative Phosphorylation (OXPHOS)?
}

Yibin Xu, ${ }^{\dagger, \nabla}$ Ding Xue, ${ }^{\dagger, \nabla}$ Armand Bankhead III, ${ }^{\ddagger, \#}$ and Nouri Neamati ${ }^{*} \dagger$

${ }^{\dagger}$ Department of Medicinal Chemistry, College of Pharmacy, Rogel Cancer Center, University of Michigan, Ann Arbor, Michigan, 48109, United States

${ }^{\ddagger}$ Department of Computational Medicine and Bioinformatics, University of Michigan Medical School, Ann Arbor, Michigan 48109, United States

${ }^{\#}$ Department of Biostatistics, University of Michigan, School of Public Health, Ann Arbor, Michigan 48109, United States

Table of contents:

Supplemental Figure 1.......................................................S2

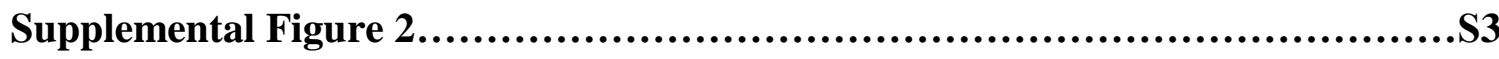

Supplemental Table 1. OXPHOS structure genes..................................S4

Supplemental Table 2. OXPHOS assembly factors and biogenesis genes .............S5

Supplemental Table 3. Canonical glycolysis genes.................................S6

Supplemental Table 4. List of physicochemical properties of 45 OXPHOS inhibitors 

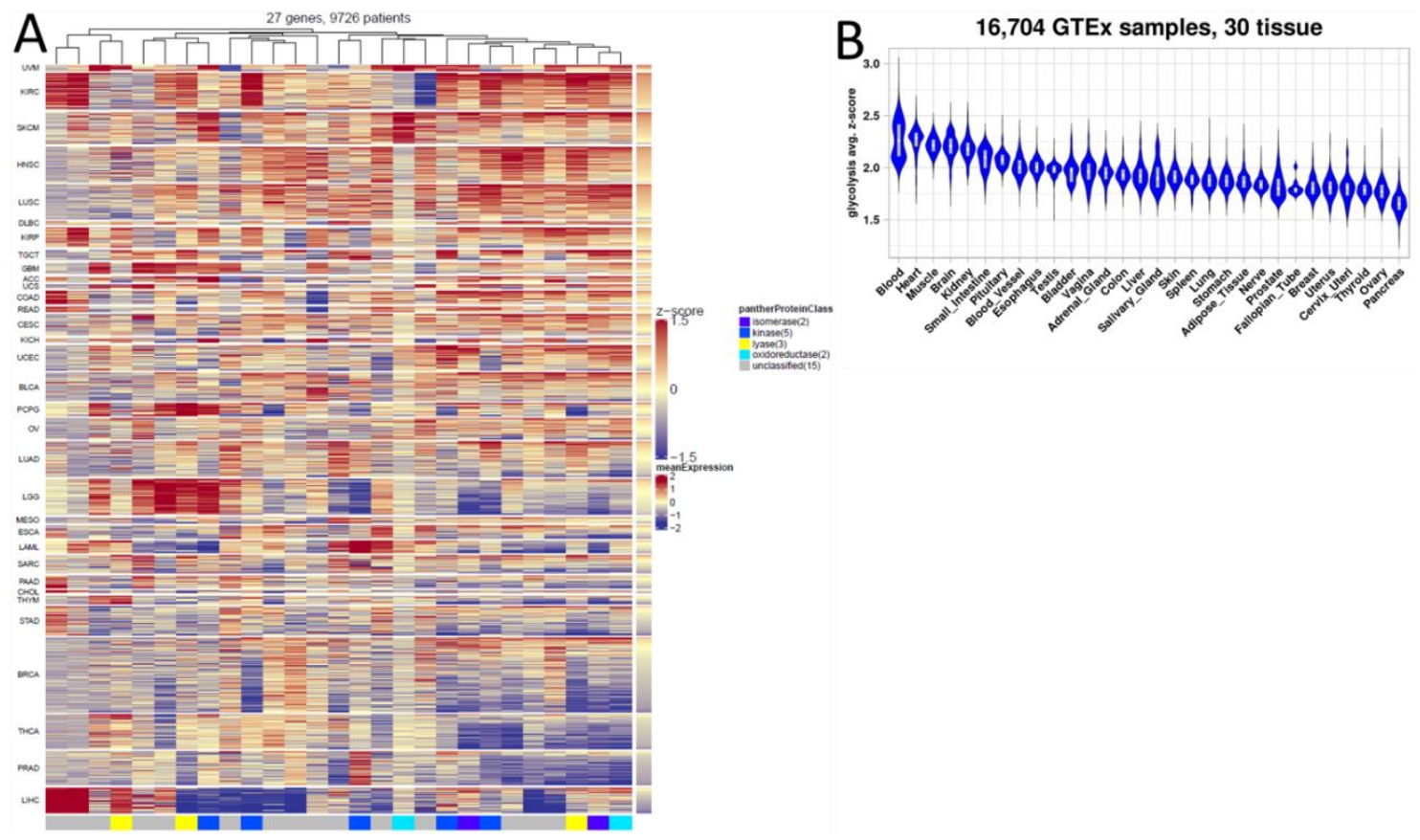

Supplemental Figure 1: (A) Pan-cancer TCGA heatmap showing z-score normalized expression of glycolysis genes with diseases ranked by average expression per disease. (B) Violin plot shows average z-score for glycolysis genes for GTEx tissue samples. Blood, heart, and muscle tissues have the highest average expression of glycolysis genes. 

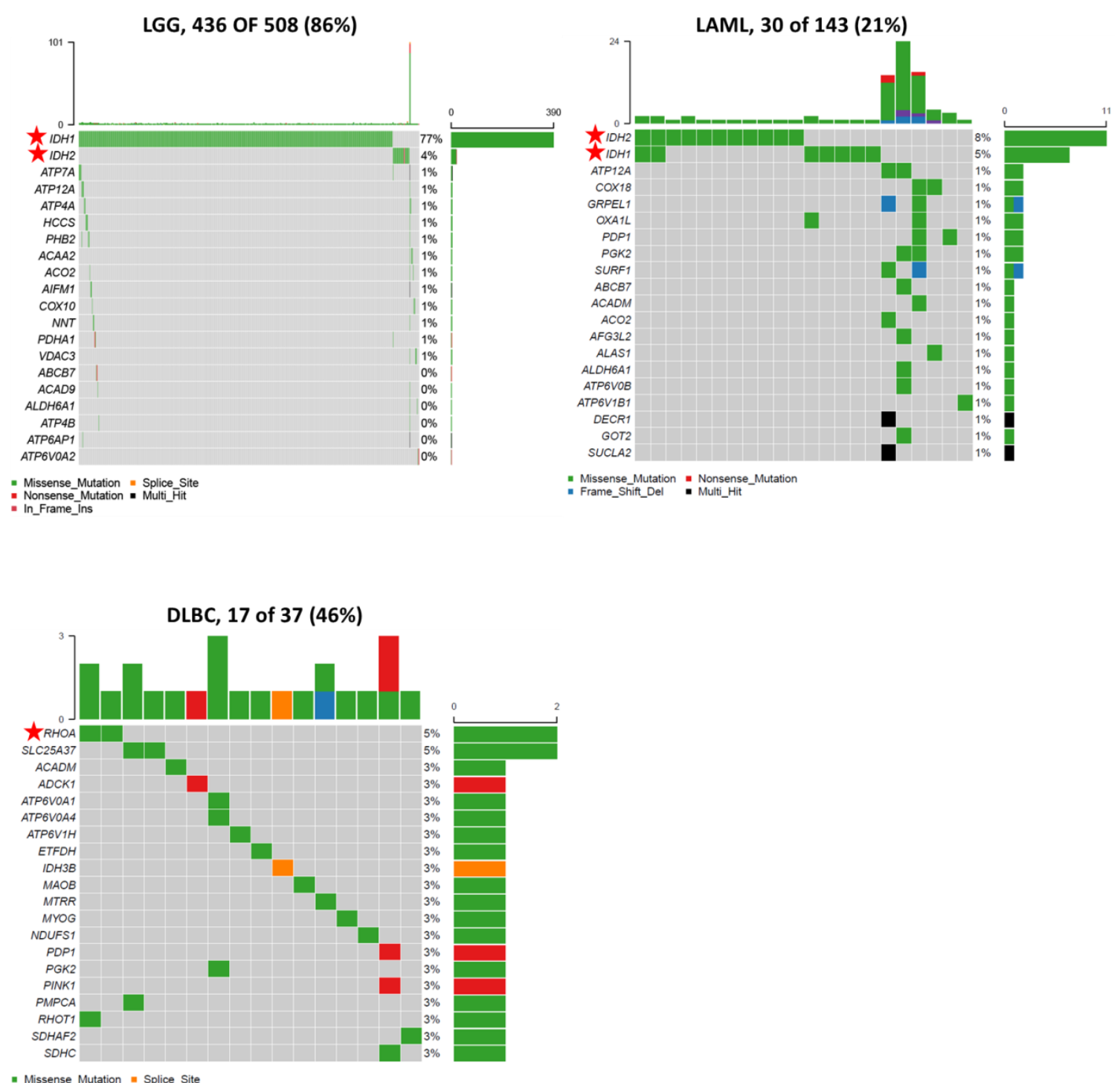

": Missense_Mutation : Splice__Site

Supplemental Figure 2: Oncoprints for lower grade glioma (LGG), acute myeloid leukemia (LAML), and lymphoid neoplasm diffuse large B-cell lymphoma (DLBC) OXPHOS gene variants. Mutations from the top 20 mutated OXPHOS genes per disease are shown. 


\section{Supplemental Table 1. OXPHOS structure genes}

\begin{tabular}{|c|c|c|c|c|c|c|c|c|}
\hline $\begin{array}{c}\text { Complex I } \\
\text { nuclear } \\
\text { genes }\end{array}$ & $\begin{array}{c}\text { Complex I } \\
\text { mtDNA } \\
\text { genes }\end{array}$ & $\begin{array}{c}\text { Complex } \\
\text { II } \\
\text { nuclear } \\
\text { genes } \\
\end{array}$ & $\begin{array}{c}\text { Complex } \\
\text { III } \\
\text { nuclear } \\
\text { genes } \\
\end{array}$ & $\begin{array}{c}\text { Complex } \\
\text { III } \\
\text { mtDNA } \\
\text { genes } \\
\end{array}$ & $\begin{array}{c}\text { Complex } \\
\text { IV } \\
\text { nuclear } \\
\text { genes } \\
\end{array}$ & $\begin{array}{c}\text { Complex } \\
\text { IV } \\
\text { mtDNA } \\
\text { genes } \\
\end{array}$ & $\begin{array}{c}\text { Complex } \\
\mathbf{V} \\
\text { nuclear } \\
\text { genes }\end{array}$ & $\begin{array}{c}\text { Complex } \\
\mathbf{V} \\
\text { mtDNA } \\
\text { genes } \\
\end{array}$ \\
\hline NDUFS1 & $\begin{array}{l}\text { MT-ND1 } \\
\end{array}$ & $\begin{array}{l}\text { SDHA } \\
\text { SDA }\end{array}$ & CYC1 & CYTB & COX4I1 & MT-CO1 & ATP5F1A & ATP6 \\
\hline NDUFS2 & MT-ND2 & SDHB & UQCRC1 & & COX4I2 & MT-CO2 & ATP5F1B & ATP8 \\
\hline NDUFS3 & MT-ND3 & SDHC & UQCRC2 & & COX5A & MT-CO3 & ATP5F1C & \\
\hline NDUFS7 & MT-ND4 & SDHD & UQCRFS1 & & COX5B & & ATP5F1D & \\
\hline NDUFS8 & MT-ND4L & & UQCRH & & COX6A1 & & ATP5F1E & \\
\hline NDUFV1 & MT-ND5 & & UQCRQ & & COX6A2 & & ATP5MC1 & \\
\hline NDUFV2 & MT-ND6 & & TTC19 & & COX6B1 & & ATP5MC2 & \\
\hline NDUFAB1 & & & UQCRB & & COX6B2 & & ATP5MC3 & \\
\hline NDUFA1 & & & UQCR10 & & COX6C & & ATP5MD & \\
\hline NDUFA2 & & & UQCR11 & & COX7A1 & & ATP5ME & \\
\hline NDUFA3 & & & & & COX7A2 & & ATP5MF & \\
\hline NDUFA5 & & & & & COX7B & & ATP5MG & \\
\hline NDUFA6 & & & & & COX7B2 & & ATP5MPL & \\
\hline NDUFA7 & & & & & COX7C & & АТР5PB & \\
\hline NDUFA8 & & & & & COX8A & & ATP5PD & \\
\hline NDUFA9 & & & & & $\operatorname{coX} 8 \mathrm{C}$ & & ATP5PF & \\
\hline NDUFA10 & & & & & & & АТР5PO & \\
\hline NDUFA11 & & & & & & & ATP5IF1 & \\
\hline \multicolumn{9}{|l|}{ NDUFA12 } \\
\hline \multicolumn{9}{|l|}{ NDUFA13 } \\
\hline \multicolumn{9}{|l|}{ NDUFB1 } \\
\hline \multicolumn{9}{|l|}{ NDUFB2 } \\
\hline \multicolumn{9}{|l|}{ NDUFB3 } \\
\hline \multicolumn{9}{|l|}{ NDUFB4 } \\
\hline \multicolumn{9}{|l|}{ NDUFB5 } \\
\hline \multicolumn{9}{|l|}{ NDUFB6 } \\
\hline \multicolumn{9}{|l|}{ NDUFB7 } \\
\hline \multicolumn{9}{|l|}{ NDUFB8 } \\
\hline \multicolumn{9}{|l|}{ NDUFB9 } \\
\hline \multicolumn{9}{|l|}{ NDUFB10 } \\
\hline \multicolumn{9}{|l|}{ NDUFB11 } \\
\hline \multicolumn{9}{|l|}{ NDUFC1 } \\
\hline \multicolumn{9}{|l|}{ NDUFC2 } \\
\hline \multicolumn{9}{|l|}{ NDUFS4 } \\
\hline \multicolumn{9}{|l|}{ NDUFS5 } \\
\hline \multicolumn{9}{|l|}{ NDUFS6 } \\
\hline NDUFV3 & & & & & & & & \\
\hline
\end{tabular}


Supplemental Table 2. OXPHOS assembly factors and biogenesis genes

\begin{tabular}{lllllll}
\hline $\begin{array}{l}\text { Complex I } \\
\text { Assembly } \\
\text { actor } \\
\text { genes }\end{array}$ & $\begin{array}{l}\text { Complex II } \\
\text { fssembly } \\
\text { factor } \\
\text { genes }\end{array}$ & $\begin{array}{l}\text { Complex III } \\
\text { Assembly } \\
\text { factor } \\
\text { genes }\end{array}$ & $\begin{array}{l}\text { Complex IV } \\
\text { Assembly } \\
\text { actor } \\
\text { genes }\end{array}$ & $\begin{array}{l}\text { Complex V } \\
\text { Assembly } \\
\text { factor } \\
\text { genes }\end{array}$ & $\begin{array}{l}\text { Coenzyme Q10 } \\
\text { (CoQ10) and } \\
\text { Cytochrome-c } \\
\text { cyt-c) Biogenesis } \\
\text { Genes }\end{array}$ & $\begin{array}{l}\text { Fe-S } \\
\text { biogenesis } \\
\text { Genes }\end{array}$ \\
\hline NDUFAF1 & SDHAF1 & BCS1L & COX10 & ATPAF1 & ADCK1 & ABCB7 \\
NDUFAF2 & SDHAF2 & UQCC1 & COX11 & ATPAF2 & ADCK2 & FLRX5 \\
NDUFAF3 & SDHAF3 & UQCC2 & COX14 & TEME70 & ADCK3 & HSPA9 \\
NDUFAF4 & SDHAF4 & UQCC3 & COX15 & OXA1L & ADCK4 & ISCA1 \\
NDUFAF5 & & & COX17 & & ADCK5 & ISCA2 \\
NDUFAF6 & & & COX18 & & COQ2 & ISCU \\
NDUFAF7 & & & COX19 & & COQ3 & LYRM4 \\
C8orf38 & & & FASTKD2 & & COQ4 & SLC25A37 \\
C20orf7 & & & FDX1 & & COQ5 & SLC25A28 \\
ACAD9 & & & LRPPRC & & COQ6 & NUBPL \\
FOXRED1 & & & OXA1L & & COQ7 & NFS1 \\
OXA1L & & PET191 & COQ8 & NFU1 \\
NUBPL & & RCF1 & & COQ9 & FXN \\
ECSIT & & SCO1 & & COQ10A & \\
NUBPL & & SCO2 & & COQ10B & \\
TEME126B & & SURF1 & CYCS & \\
& & TACO1 & HCCS & \\
& & COA5 & PDSS1 & \\
& & & PDSS2 & \\
\hline
\end{tabular}


Supplemental Table 3. Canonical glycolysis genes

\begin{tabular}{lll}
\hline Gene & Entrez & HGNC ID \\
\hline ADPGK & 83440 & HGNC: 25250 \\
ALDOA & 226 & HGNC: 414 \\
ALDOB & 229 & HGNC: 417 \\
ALDOC & 230 & HGNC: 418 \\
BPGM & 669 & HGNC: 1093 \\
ENO1 & 2023 & HGNC: 3350 \\
ENO2 & 2026 & HGNC: 3353 \\
ENO3 & 2027 & HGNC: 3354 \\
FOXK1 & 221937 & HGNC: 23480 \\
FOXK2 & 3607 & HGNC: 6036 \\
GAPDH & 2597 & HGNC: 4141 \\
GAPDHS & 26330 & HGNC: 24864 \\
GCK & 2645 & HGNC: 4195 \\
GPI & 2821 & HGNC: 4458 \\
HK1 & 3098 & HGNC: 4922 \\
HK2 & 3099 & HGNC: 4923 \\
HK3 & 3101 & HGNC:4925 \\
PFKL & 5211 & HGNC: 8876 \\
PFKM & 5213 & HGNC: 8877 \\
PFKP & 5214 & HGNC: 8878 \\
PGAM1 & 5223 & HGNC: 8888 \\
PGAM2 & 5224 & HGNC: 8889 \\
PGK1 & 5230 & HGNC: 8896 \\
PGM2L1 & 283209 & HGNC: 20898 \\
PKLR & 5313 & HGNC: 9020 \\
PKM & 5315 & HGNC: 9021 \\
TPI1 & 7167 & HGNC: 12009 \\
\hline & & \\
\hline
\end{tabular}


Supplemental Table 4. List of physicochemical properties of 45 OXPHOS inhibitors

\begin{tabular}{|c|c|c|c|c|c|c|}
\hline Name & $\begin{array}{c}\text { H- } \\
\text { bond } \\
\text { donor }\end{array}$ & $\begin{array}{l}\text { H-bond } \\
\text { acceptor }\end{array}$ & Fsp $^{3}$ & cLogP & TPSA & MW \\
\hline Metformin & 4 & 5 & 0.5 & -0.92 & 88.99 & 129.17 \\
\hline Phenformin & 5 & 5 & 0.2 & 0.833 & 97.78 & 205.27 \\
\hline $\begin{array}{c}\text { IM156 } \\
\text { (HL156A) }\end{array}$ & 4 & 6 & 0.38 & 3.10 & 84.23 & 315.30 \\
\hline BAY 87-2243 & 0 & 7 & 0.38 & 6.25 & 85.34 & 525.54 \\
\hline IACS-010759 & 0 & 8 & 0.36 & 5.24 & 116.24 & 562.57 \\
\hline VLX600 & 2 & 6 & 0.12 & 2.97 & 91.74 & 317.36 \\
\hline Lonidamine & 1 & 3 & 0.07 & 4.40 & 55.12 & 321.16 \\
\hline Atovaquone & 1 & 3 & 0.27 & 5.00 & 54.37 & 366.84 \\
\hline AG311 & 3 & 4 & 0.06 & 3.85 & 93.61 & 321.4 \\
\hline Rotenone & 0 & 6 & 0.35 & 3.32 & 63.22 & 394.42 \\
\hline Mubritinib & 0 & 4 & 0.24 & 5.96 & 65.97 & 468.48 \\
\hline $\begin{array}{c}\text { Carboxyamido } \\
\text { triazole }\end{array}$ & 2 & 5 & 0.06 & 4.16 & 116.89 & 424.67 \\
\hline
\end{tabular}




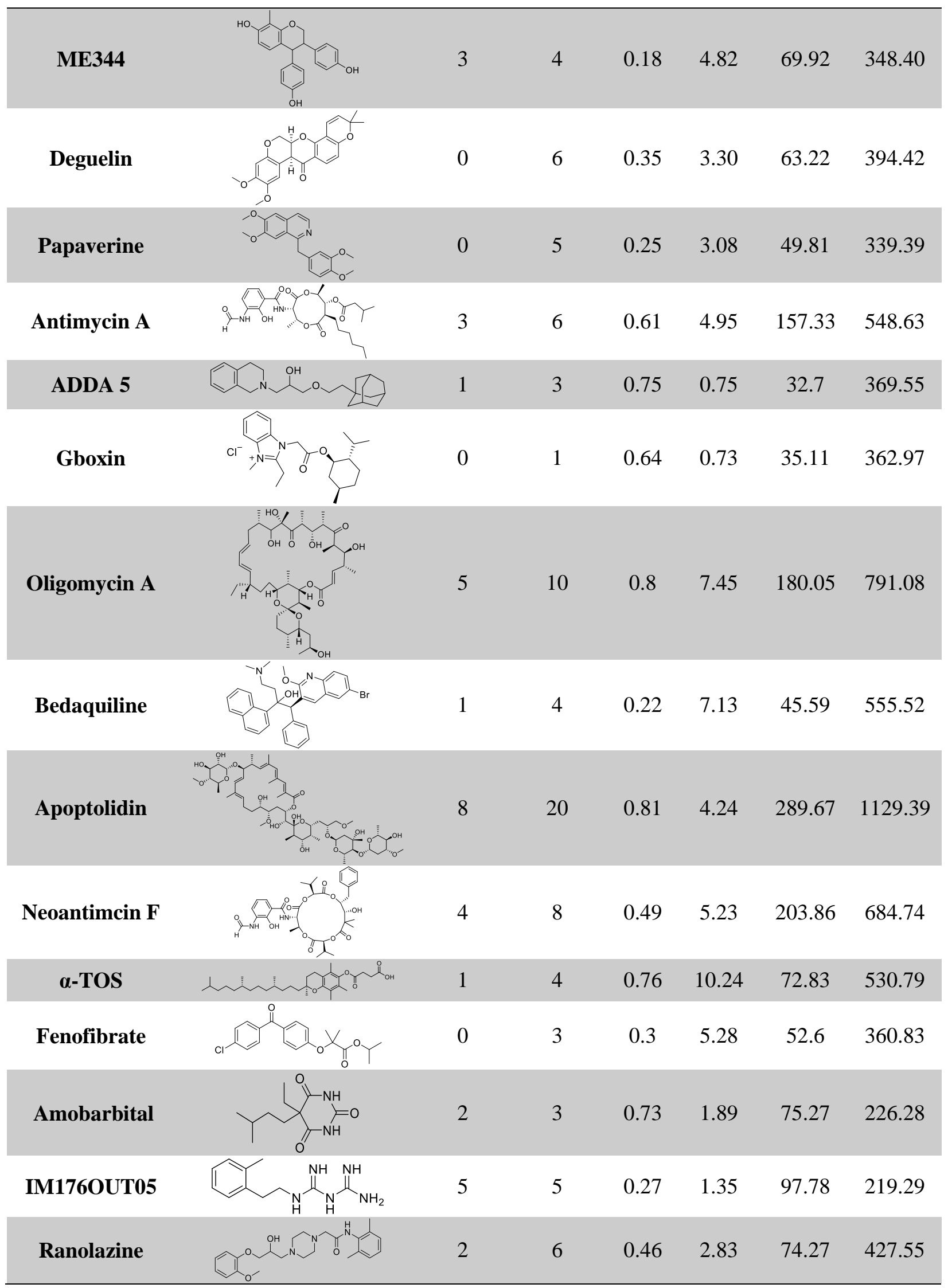




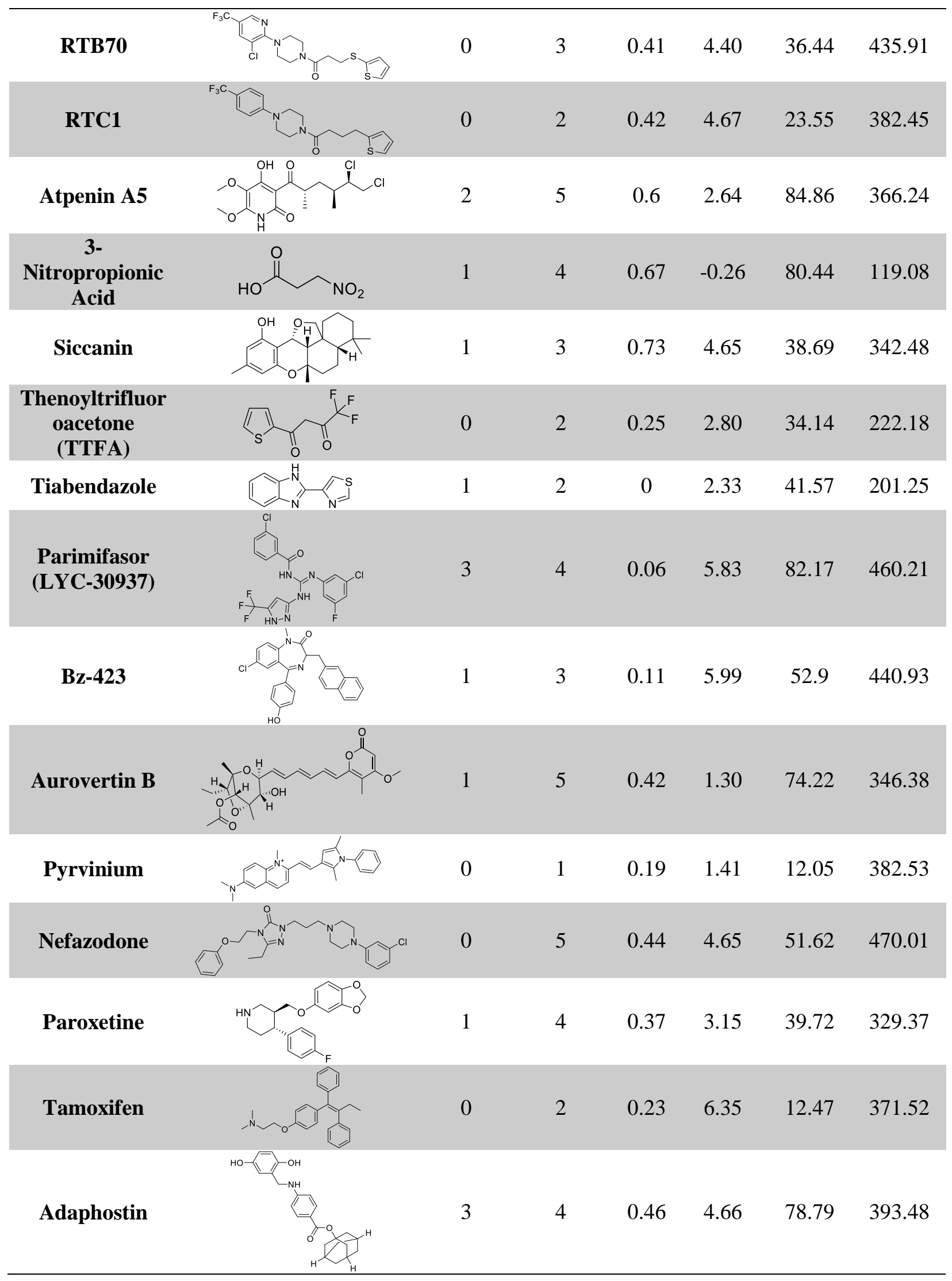




Pamellarin D

